\title{
Vesiko-urethrale Dysfunktion
}

\begin{abstract}
Vesiko-urethrale Dysfunktion als Ausdruck aller Funktionsstörungen am unteren Harntrakt beinhaltet eine Vielfalt an Symptomen und patho-physiologischen Mechanismen. Viele Zusammenhänge sind dabei nach wie vor nicht bekannt, so z. B. die Verknüpfung von Blaseninstabilität - Compliance - Kontraktilität (Miktionsdruck). Strukturelle, funktionelle und metabolische Veränderungen des Detrusors spielen ebenso eine Rolle wie neurale Aspekte, insbesondere die koordinierte Steuerung der Füllungsund Entleerungsphase. Durch international akzeptierte, standardisierte Begriffe und Untersuchungen, die durch die International Continence Society mit Konzepten für eine allgemein akzeptierte Klassifikation der vesiko-urethralen Dysfunktion erstellt wurden, ist es möglich, klinische Standards für die Diagnostik und Therapie zu erarbeiten.
\end{abstract}

Einige diagnostische und therapeutische Konzepte für neurogene wie nicht-neurogene funktionelle Störungen sollen im folgenden dargestellt werden. Nicht $z u-$ letzt deshalb, da es gerade bei neurogenen Funktionsstörungen gelungen ist, Risikofaktoren zu definieren, die, wenn sie frühzeitig erkannt werden, die Lebenserwartung dieser Patienten wesentlich verbessern, wenn nicht sogar der allgemeinen Lebenserwartung gleichsetzen.

Neuromodulation und Neurostimulation sind neue Konzepte in der Behandlung funktioneller Störungen mit vielversprechenden Behandlungsergebnissen. Unverändert bleibt die Zielsetzung der Erhaltung der Nierenfunktion, der Schaffung eines Niederdrucksystems am unteren Harntrakt mit Kontinenz während der Speicherphase. Beispielhaft wird dies in der Arbeit von M. Stöhrer dargelegt, der durch Autoaugmentation der Blase in speziellen Fällen eine Niederdrucksituation mit vergrößerter Kapazität und Kontinenz herstellen konnte. Vielfach ist bei neurogenen Störungen eine enge Zusammenarbeit zwischen Urologen, Neurologen, Rehabilitationsmedizinern, Orthopäden und Neurochirurgen notwendig. Die Schaffung der Subspezialität „Neuro-Urologie" ist Ausdruck der Bemühungen um urologische Behandlungskonzepte bei neurogenen Störungen klinisch umzusetzen.

Da die Symptomatik verschiedener Funktionsstörungen recht unspezifisch ist, um die zugrunde liegende pathophysiologische oder-morphologische Störung zu identifizieren, ist es wichtig, ein klares Konzept für die Diagnostik zu erarbeiten. Nur unter Einschluß der urodynamischen Diagnostik, ist eine vesiko-urethrale Dysfunktion zu definieren. Das Ausmaß und der Umfang dieser urodynamischen Diagnostik ist jedoch unterschiedlich und sollte an die Symptomatik und die zugrunde liegende Ätiologie angepaßt werden. Bei neurogenen Blasenstörungen ist die videourodynamische Diagnostik zwingend. Eine Therapie ex iuvantibus darf bei einer neurogenen Funktionsstörung nicht durchgeführt werden und bei einer funktionellen, nicht-neurogenen Störung nur ganz kurzfristig.

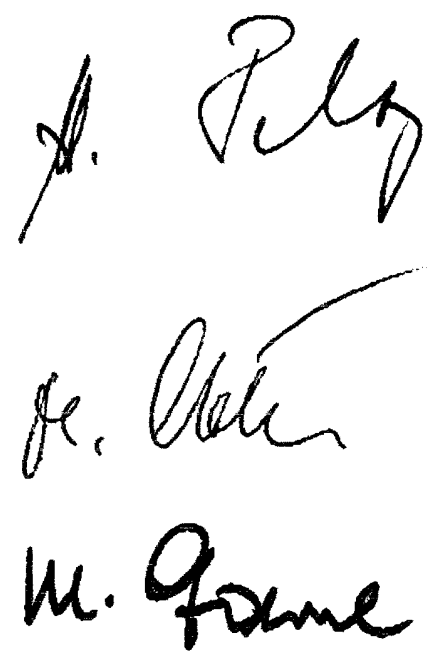

